
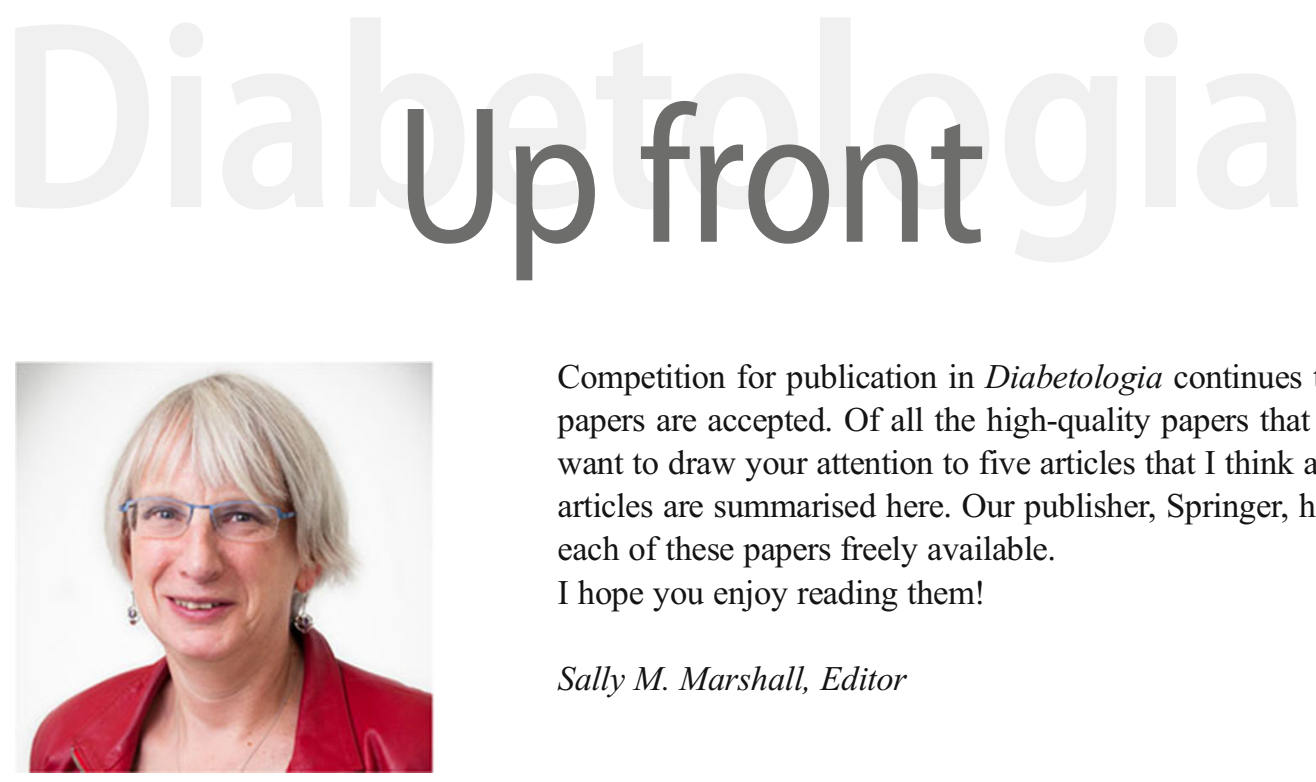

Competition for publication in Diabetologia continues to grow, and less than $20 \%$ of papers are accepted. Of all the high-quality papers that appear in this month's issue I want to draw your attention to five articles that I think are particularly interesting. The articles are summarised here. Our publisher, Springer, has kindly made the full text of each of these papers freely available.

I hope you enjoy reading them!

Sally M. Marshall, Editor

\section{Advances in drug discovery for human beta cell regeneration}

Esra Karakose, Courtney Ackeifi, Peng Wang, Andrew F. Stewart

Reduced numbers of insulin-secreting beta cells underlie both type 1 and type 2 diabetes. Conversely, residual beta cells are present in people with type 2 diabetes, and even after 50 years of type 1 diabetes. The current diabetes armamentarium includes insulin replacement and drugs that encourage residual beta cells to secrete more insulin and/or enhance sensitivity to insulin. Beta cell replacement, via transplantation of whole pancreas, cadaveric islets or stem cell-derived beta cells, is another approach. However, as reviewed in this issue by Karakose et al (https://doi.org/10.1007/s00125-018-4639-6), a simpler and more direct alternative would be to expand the numbers of residual beta cells in people with diabetes. Although human beta cells have long been viewed as terminally differentiated and irreversibly quiescent, this notion is changing owing to the recent discovery of pharmacological tools that can induce adult human beta cells to replicate. The authors review progress in this area, and outline remaining obstacles to bringing these novel therapies to patients.

(t) The figures from this review are available as a downloadable slideset.
Systematic review assessing the effectiveness of dietary intervention on gut microbiota in adults with type 2 diabetes

David Houghton, Timothy Hardy, Christopher Stewart, Linda Errington, Christopher P. Day, Michael I. Trenell, Leah Avery

In type 2 diabetes, treatment variability and disease progression remain poorly understood. However, mechanistic preclinical studies indicate that the gut microbiome may be involved. In this issue, Houghton et al (https://doi.org/10.1007/ s00125-018-4632-0) report the results of a systematic review, which included eight eligible studies. They found that dietary modification and various pre-, pro- and symbiotic supplements are able to modulate the composition of the gut microbiome and improve glucose control in people with type 2 diabetes. The findings provide important insight but also highlight the need for further well-conducted interventional studies in humans using standardised approaches to allow direct comparisons to be made. The authors note that a deeper understanding of the interaction between the gut microbiome and the pathophysiology of type 2 diabetes will help to lay the foundations to translate preclinical data into clinical practice. If successful, manipulating the microbiome may provide another pathway for the management of type 2 diabetes, enabling a personalised lifestyle approach. 
Reduced risk of heart failure with intensified multifactorial intervention in individuals with type 2 diabetes and microalbuminuria: 21 years of follow-up in the randomised Steno-2 study

Jens Oellgaard, Peter Gcede, Peter Rossing, Rasmus Rørth, Lars Køber, Hans-Henrik Parving, Oluf Pedersen

In type 2 diabetes, heart failure is a common, late stage complication that is associated with a high mortality rate that, until recently, has been investigated little. In this issue, Oellgaard and colleagues (https://doi.org/10.1007/s00125-018-4642-y) present the results of a post hoc analysis from 21.2 years' follow-up from the Steno-2 study of intensified vs conventional multifactorial intervention in high-risk individuals. Heart failure hospitalisations were as frequent as atherosclerotic cardiovascular disease and were significantly reduced by $70 \%$ in the intensive therapy group. The composite endpoints, heart failure hospitalisation or cardiovascular death and heart failure or all-cause mortality, were significantly reduced by $62 \%$ and $49 \%$, respectively. Incident heart failure was associated with, but not explained by, prior myocardial infarction. Along with conventional risk factors, elevated plasma Nterminal pro-B-type natriuretic peptide (NT-proBNP) at baseline was associated with heart failure and, in the conventional therapy group, an increase in NT-proBNP during the initial 2 years was associated with a poorer outcome. The authors suggest that caregivers should recommend intensified multifactorial intervention to patients with type 2 diabetes to reduce the risk of heart failure.

\section{Multiplex proteomics for prediction of major cardiovascular events in type 2 diabetes}

Christoph Nowak, Axel C. Carlsson, Carl Johan Östgren, Fredrik H. Nyström, Moudud Alam, Tobias Feldreich, Johan Sundström, Juan-Jesus Carrero, Jerzy Leppert, Pär Hedberg, Egil Henriksen, Antonio C. Cordeiro, Vilmantas Giedraitis, Lars Lind, Erik Ingelsson, Tove Fall, Johan Ärnlöv

There is a need to identify those individuals with type 2 diabetes who are at high risk of heart attack and stroke so that they can receive targeted prevention interventions. In this issue, Nowak et al (https://doi.org/10.1007/s00125-018$4641-\mathrm{z}$ ) at the Karolinska Institute in Sweden assessed whether blood-borne proteins with presumed roles in inflammation and cardiovascular disease might predict the risk of cardiovascular events in type 2 diabetes. Using an 80-protein multiplex assay, the authors replicated four previously described associations and discovered four novel associations. The addition of protein biomarkers to an available risk model improved the prediction of cardiovascular events in people with type 2 diabetes. The authors conclude that, pending further evaluation in a clinical context, these results suggest that targeted multi-protein assays can improve the risk assessment of serious cardiovascular events in type 2 diabetes.

Effects of acute NEFA manipulation on incretin-induced insulin secretion in participants with and without type 2 diabetes

Brenno Astiarraga, Valéria B. Chueire, Aglécio L. Souza, Ricardo Pereira-Moreira, Sarah Monte Alegre, Andrea Natali, Andrea Tura, Andrea Mari, Ele Ferrannini, Elza Muscelli

In type 2 diabetes, stimulation of insulin secretion by the entry of glucose into the digestive system (i.e. the incretin effect) is impaired. Recent experimental evidence suggests that NEFA might interfere with incretin function. In this issue, Astiarraga, Chueire et al (https://doi.org/10.1007/s00125-018-4633-z) tested this hypothesis by exposing individuals without diabetes to an acute rise in NEFA and by lowering NEFA in participants with type 2 diabetes. The results indicate the presence of a clear asymmetry: while elevation of NEFA disrupted the incretin effect in those without diabetes, lowering NEFA in individuals with type 2 diabetes had no effect. Neither beta cell sensitivity to glucose nor plasma incretin hormone concentrations were altered by NEFA manipulation. Whilst modest elevations of NEFA typically observed in type 2 diabetes are unlikely to be solely responsible for reduced beta cell sensitivity to incretins, they may have some role in disease progression; however, this remains to be demonstrated conclusively.

All text supplied by the authors. 